\title{
Lumen
}

Selected Proceedings from the Canadian Society for Eighteenth-Century Studies

\section{Le monde du spectacle et le spectacle du monde : la nouvelle esthétique dramatique au XVIII ${ }^{\mathrm{e}}$ siècle}

\section{Romira Worvill}

Volume 22, 2003

URI : https://id.erudit.org/iderudit/1012262ar

DOI : https://doi.org/10.7202/1012262ar

Aller au sommaire du numéro

Éditeur(s)

Canadian Society for Eighteenth-Century Studies / Société canadienne d'étude du dix-huitième siècle

ISSN

1209-3696 (imprimé)

1927-8284 (numérique)

Découvrir la revue

Citer cet article

Worvill, R. (2003). Le monde du spectacle et le spectacle du monde : la nouvelle esthétique dramatique au XVIII ${ }^{\mathrm{e}}$ siècle. Lumen, 22, 117-134.

https://doi.org/10.7202/1012262ar

Copyright (c) Canadian Society for Eighteenth-Century Studies / Sociéte canadienne d'étude du dix-huitième siècle, 2003
Ce document est protégé par la loi sur le droit d'auteur. L'utilisation des services d'Érudit (y compris la reproduction) est assujettie à sa politique d'utilisation que vous pouvez consulter en ligne.

https://apropos.erudit.org/fr/usagers/politique-dutilisation/ 


\section{Le monde du spectacle et le spectacle du monde: la nouvelle esthétique dramatique au $\mathrm{XVIII}^{\mathrm{e}}$ siècle}

Normalement, au théâtre, c'est la réalisation sur scène de pièces innovatrices qui sert à déclencher une modification des normes esthétiques en vigueur. Mais il n'en est pas ainsi pour le drame et la tragédie bourgeoise en France au XVIII siècle. A cette époque, l'esthétique naissante s'élabore d'abord dans les écrits de critiques et d'écrivains qui, dans un premier temps, cherchent à exposer les faiblesses (selon eux) de la tragédie néoclassique et, ensuite, à communiquer à leurs contemporains l'idée d'une nouvelle forme dramatique plus apte à exprimer le vécu de leur époque. Les frondeurs se trouvent ainsi devant une tâche peu facile, celle d'évoquer pour leurs lecteurs une forme artistique dont ils n'ont pas encore d'exemple convaincant en français. L'effet visé par les partisans du théâtre bourgeois est l'illusion telle qu'elle sera définie par Gotthold Ephraïm Lessing dans son Laocoon de 1766. Dans la préface de cet ouvrage, Lessing invoque la comparaison classique entre poésie et peinture. Il affirme que les deux arts ont ceci de commun qu'ils «stellen uns abwesende Dinge als gegenwärtig, den Schein als Wirklichkeit vor; beide täuschen, und beider Täuschung gefällt ${ }^{1} »$. Or la création de ce genre d'illusion n'avait pas été l'objectif du théâtre néoclassique en France, conçu dans le cadre d'une esthétique qui met l'accent sur la subjugation heureuse des difficultés inhérentes à la forme plutôt que sur la représentation mimétique. Pour expliquer aux lecteurs le caractère unique du théâtre à créer, les commentateurs commencent donc à faire appel à deux figures qui, passant d'un écrivain à l'autre, finissent par devenir les lieux communs de la nouvelle esthétique théâtrale. Ces

1 Gotthold Ephraïm Lessing, Werke, édition de Herbert G. Göpfert, Munich, Carl Hanser Verlag, 1970-1979, 8 tomes, t. VI, p. 9. Ces deux arts «nous présentent des choses absentes comme si elles étaient présentes, des apparences comme si elles étaient des réalités. Ils nous trompent, et cette déception plaît.» 
figures sont le "poète disparu» et le "spectateur caché» qui, ensemble, constituent les deux pôles de la transformation à effectuer; l'œuvre et la réception de l'œuvre. Dans cet article, nous allons commencer par démontrer l'omniprésence de ces deux topoï dans la théorie du théâtre, pour parler ensuite des liens de parenté qui semblent exister entre ces images et le discours contemporain dans les domaines de la peinture et de la science.

La première de ces images à s'imposer - celle du poète disparu surgit d'abord sous la plume d'Antoine Houdar de La Motte, poète reconnu et partisan des Modernes. Au début, l'idée du "poète invisible» entre presque par hasard dans ses œuvres, où on la trouve reliée à une critique des excès du langage figuré. Les poètes lyriques, dit La Motte dans la préface d'un recueil de ses odes (1707), «peuvent et doivent même étaler toutes les richesses de la poésie», car ils parlent comme «des poètes de profession ${ }^{2} »$. Les poètes dramatiques, par contre, «ne font point parler des poètes, mais des hommes ordinaires». Par conséquent, «ils doivent toujours être en garde contre l'excès de l'expression» et «ils ne doivent qu'exprimer les sentiments qui conviennent à leurs acteurs, et prendre pour cela les tours et les termes que la passion offre le plus naturellement». La réaction négative que lui attire cette critique du discours figuré sur la scène (et notamment de la part de Boileau) ne sert qu'à consolider ses convictions ${ }^{3}$. Puis, en 1730 , il revient à la charge dans une série d'essais commentant ses propres tragédies. Poursuivant la logique du topos jusqu'à sa conclusion inévitable, il se fonde sur elle pour revendiquer l'abandon de l'alexandrin en faveur d'un discours en prose, car, dit-il, la forme même des vers, la mesure et la rime attirent l'attention du spectateur sur le langage en tant que langage, dévoilant ainsi la véritable source de la parole, le poète. De là, il n'y a qu'un pas pour englober dans sa critique d'autres éléments clés de l'esthétique néoclassique: sentences, monologues, récits, confidents et expositions se présentent désormais à La Motte comme autant de conventions qui dénoncent la pièce comme artifice. La figure du poète disparu devient la clé de voûte

2 Antoine Houdar de La Motte, CEuvres, Paris, 1753-1754, 9 tomes et supplément, t. I, p. 26.

3 Boileau attaque les idées de La Motte dans la onzième de ses Réflexions sur Longin, publiée en 1710. La Motte y revient dans sa Réponse à M. Despréaux, qui parut en 1713-1714, soit deux années après la mort de son illustre adversaire (OEuvres, op. cit., t. V, 83-99). Le commentaire de Boileau met au jour le gouffre qui commence à s'ouvrir entre la conception néoclassique de la parole théâtrale et la place qui lui sera accordée dans la nouvelle esthétique. 
d'une conception du théâtre qui prend comme objectif primaire la création de l'illusion. Selon La Motte, le dramaturge doit

ajuster tout pour les effets qu'[il] a en vue, sans laisser apercevoir de dessein, de manière enfin que le spectateur voie toujours une action, et ne sente jamais un ouvrage: car dès que l'auteur prend ses avantages aux dépens de la moindre vraisemblance, il les peut perdre par cela même. L'illusion cesse. On ne voit plus que le poète au lieu des personnages; et on lui tient d'autant moins compte de ses beautés, qu'il ne les amène qu'en sortant du naturel et des convenances. [...] En un mot, tout est art du côté de celui qui arrange une action théâtrale; mais rien ne le doit paraître à celui qui la voit ${ }^{4}$.

Cette image du poète disparu n'est pas nouvelle. En l'invoquant, La Motte s'appuie sur l'autorité d'Horace qui aurait dit, dans une de ses satires: «l'éloge du poète épique est que, si l'on rompt la mesure de ses vers, on retrouve toujours les membres épars d'un poète, l'éloge de l'auteur dramatique, c'est qu'en rompant de même les mesures, le Poète disparaisse et ne laisse voir que le personnage ${ }^{5} »$. Il s'agit, en l'occurrence, de quelques vers tirés du quatrième poème de son premier livre de satires, où Horace fait appel à cette idée pour caractériser son propre style, style qu'il considère peu poétique ${ }^{6}$. La Motte n'hésite pas à s'emparer de ce topos pour justifier un discours théâtral en prose, créé dans le but de faire illusion aux spectateurs; sous cette forme, il fera fortune auprès des écrivains de la génération suivante, engagés à prolonger l'attaque contre la tragédie néoclassique et à lancer de nouveaux genres.

Dès 1748 et la parution de ses Bijoux indiscrets, Diderot l'invoque pour critiquer la tragédie cornélienne:

Au moins, madame, répliqua Sélim, vous ne nierez pas que, si les épisodes nous tirent de l'illusion, le dialogue nous y ramène. Je ne vois personne qui l'entende comme nos tragiques.

-Personne n'y entend donc rien, reprit Mirzoza. L'emphase, l'esprit et le papillotage qui y règnent sont à mille lieues de la nature. C'est en vain que

4 Ibid., t. IV, p. 284-85

5 Ibid., t. IV, p. 414-15.

6 Voir Horace, Eupolis atque Cratinus Aristophanesque pætx, vers 59-62 dans The Satires and Epistles of Horace, traduction de Smith Palmer Bovie, Chicago, University of Chicago Press, 1996 (1959), p. 53-54. 
l'auteur cherche à se dérober; mes yeux percent, et je l'aperçois sans cesse derrière ses personnages ${ }^{7}$.

En 1760, Beaumarchais y fait appel pour louer la réussite du dialogue en prose de Sedaine dans le Philosophe sans le savoir:

C'est dans le salon de Vanderk quej'ai tout à fait perdu de vue Préville et Brisard, pour ne voir que le bon Antoine et son excellent maître, et m'attendrir véritablement avec eux. Croyez-vous que cela me fût arrivé de même, s'ils m'eussent récité des vers? Non seulement j'aurais retrouvé les acteurs dans les personnages, mais qui pis est, à chaque rime j'aurais aperçu le poète dans les acteurs ${ }^{8}$.

Mercier, dans son Nouvel essai sur l'art dramatique (1773), se sert des mêmes termes pour caractériser le style qui convient au drame:

Demandez des vers à un poète épique, c'est le poète qui parle, qui embouche la trompette; mais dans le drame c'est le personnage seul qui doit paraître, et non l'auteur. [...] Ce n'est donc pas le langage des Dieux, mais le langage des hommes qu'il faut produire sur le théâtre?.

Il y reviendra dans son Nouvel examen de la tragédie française, critiquant le poète «qui veut toujours être aperçu et se mêler parmi ses personnages ${ }^{10} »$. Enfin Melchior Grimm, ami et collaborateur de Diderot, reprend la même pensée dans un compte rendu de sa Correspondance littéraire:

Dans la poésie épique, c'est le droit du poète de se montrer toujours à côté de ses héros, c'est lui qui parle lors même qu'il fait parler les autres; mais dans la

7 Diderot, CEuvres, édition de Laurent Versini, Paris, Robert Laffont, 5 tomes, 1996, t. II, p. 124. Cette image revient souvent dans les écrits sur le théâtre de Diderot. Par exemple: «Dans une représentation dramatique, il ne s'agit non plus du spectateur que s'il n'existait pas. Y a-t-il quelque chose qui s'adresse à lui? L'auteur est sorti de son sujet, l'acteur entraîné hors de son rôle. Ils descendent tous les deux du théâtre. Je les vois dans le parterre» (ibid., t. IV, p. 1145).

8 Beaumarchais, CFuvres, édition de Pierre Larthomas, Paris, Gallimard, coll. «Bibliothèque de la Pléiade», 1988, p. 133.

9 Louis Sébastien Mercier, Du théâtre, ou Nouvel essai sur l'art dramatique, Amsterdam, 1773, p. 269 et 301.

10 Louis Sébastien Mercier, De la littérature et des littérateurs, suivi d'un nouvel examen de la tragédie française, Yverdon, 1778; Genève, Slatkine reprints, 1970, p. 113. 
poésie dramatique, le sublime de son art, c'est de ne se jamais rappeler à l'idée du spectateur ${ }^{11}$.

Notons en particulier le glissement qui s'opère dans tous ces extraits d'une impression auditive vers une image visuelle. Cette tendance, déjà évidente chez La Motte, est flagrante dans le texte de Diderot qui nous propose une espèce de paradoxe, celui d'un discours saisi par l'oreille qui expose le poète à la vue des spectateurs. On entend des paroles, mais on «voit» un auteur. Ce phénomène n'est pas sans intérêt, car il met à nu la transition en train de s'effectuer entre un théâtre de la parole et celui de l'image. Les termes de la discussion trahissent le profond désir chez ces théoriciens de substituer au monde virtuel créé par les mots une véritable illusion du réel. Il faut bannir le poète de la scène pour que son monde fictif puisse seul en prendre possession.

L'importance de la vue pour l'esthétique en évolution ressort encore plus clairement de la deuxième figure que nous voulons évoquer, celle du spectateur caché. Cette image paraît d'abord dans le prologue de la première tragédie bourgeoise française, Silvie, créée par Paul Landois et représentée à la Comédie-Française en $1741^{12}$. La forme de la pièce manifeste une intention très délibérée de transgresser de nombreux principes du classicisme. Le prologue en est comique et semble viser l'anéantissement des frontières entre scène et auditoire, car les membres $\mathrm{du}$ public se trouvent en présence d'un groupe de personnages qui, comme eux, sont venus assister à la pièce controversée. En attendant le commencement du spectacle, les personnages se disputent, incapables de se mettre d'accord pour nommer "tragédie» une pièce en un acte et en prose qui met en scène des gens ordinaires et dans laquelle il est question de choses aussi banales que meubles et vêtements. Pour mieux

11 Correspondance littéraire, édition de Maurice Tourneux, Paris 1877-1882, 16 tomes, t. VIII, p. 461. Grimm analyse une pièce en vers de Jean-François La Harpe. N.C.J. Trublet, critique littéraire de l'époque, affirme que c'est La Motte qui a mis à la mode ce contraste capital entre poète et personnage. Reproduisant dans un recueil de ses essais les arguments en faveur du discours en prose qu'il avait publiés dans le Mercure au moment de la querelle de 1730, il note que «le prétendu paradoxe de M. de la Motte sur les tragédies en prose, n'en est presque plus un». Trublet insiste encore sur la distinction entre auteur et personnage, entre "l'homme de lettres et l'homme» (Essais sur divers sujets de littérature et de morale, Paris, 1760; Genève, Slatkine reprints, 1968, 4 tomes, t. IV, p. 399-400).

12 La pièce eut deux représentations à la Comédie-Française en 1741 et fut publiée par Prault Fils en 1742, sans nom d'auteur. Elle fut par la suite attribuée à Paul Landois, auteur de plusieurs articles sur la peinture parus dans l'Encyclopédie. 
expliquer les effets envisagés par l'auteur, le commandeur, qui est son ami, propose de considérer la pièce du point de vue d'un spectateur caché. Il demande à l'un de ses interlocuteurs, le chevalier, ce que lui ferait si, par hasard, se retrouvant à côté des appartements royaux, il apercevait, à travers quelques ouvertures ignorées, des courtisans qui complotaient contre le prince et tiraient le poignard pour le tuer. Le chevalier répond sans hésiter qu'il enfoncerait la porte et tomberait sur les traîtres, l'épée à la main. Le commandeur approuve, puis il lui propose de comparer la scène qu'il vient d'imaginer avec une représentation de la même situation selon les conventions de la tragédie classique. Quel effet cette scène aurait-elle sur lui, surtout s'il était étranger et ignorait tout des conventions de la représentation tragique en France? Cette fois le chevalier ne sait quoi dire, mais le commandeur imagine facilement sa réaction:

Ton courage et ton zèle ont dicté ta réponse dans le premier cas. Dans le second, sans crainte de passer pour un sujet téméraire, ce même zèle te ferait faire des vœux au ciel pour qu'il fît recouvrer la raison à ton prince; car tu dirais: «Si ce n'est point une mascarade, dans laquelle il s'est engagé, il est fou; et sa cour est folle ${ }^{13} . »$

Son exemple montre que les discours et le jeu de la tragédie classique sont tellement éloignés de ce qu'on voit et entend autour de soi qu'un spectateur n'arriverait pas, à partir du simple témoignage de ses sens, à relier l'action de la scène avec son expérience du milieu social où il se trouve.

En 1748, Diderot reprend l'anecdote exploitée par Landois dans le chapitre des Bijoux indiscrets déjà cité. Il y a quelques différences de détail imposées par le cadre du roman, mais, dans le fond, il s'agit du même exemple employé aux mêmes fins, la remise en question d'une forme dramatique qui dépend d'un système de langage et de gestes de conven$\operatorname{tion}^{14}$. Dix ans plus tard, Diderot reviendra à ce topos pour structurer ses ouvrages clés sur le théâtre, le Fils naturel et les Entretiens sur le Fils naturel, mais dans une perspective un peu différente. Il l'exploite cette fois pour faire valoir l'impact que pourraient avoir des discours en prose, accompagnés d'un jeu naturel et soutenus pendant toute la pièce. Le narrateur, Moi, persuade Dorval de le cacher dans le coin d'un salon d'où il

13 Henry Carrington Lancaster, The First French «tragédie bourgeoise»: Silvie, Attributed to Paul Landois, Baltimore, coll. «The Johns Hopkins Studies in Romance Literatures and Languages», 48, 1954, p. 10-11.

14 Voir Diderot, CEuvres, op. cit., t. II, p. 125. 
observera, à l'insu des participants, la reconstitution dramatique d'une expérience familiale. La réaction de ce témoin caché est tout le contraire de celle des spectateurs des deux premières versions de ce topos. Loin de penser qu'il assiste à une mascarade, ce spectateur est saisi au point d'oublier presque qu'il s'agit d'une scène de théâtre. Il constate:

L'histoire de Dorval était connue dans le pays. La représentation en avait été si vraie qu'oubliant en plusieurs endroits que j'étais spectateur, et spectateur ignoré, j'avais été sur le point de sortir de ma place, et d'ajouter un personnage réel à la scène ${ }^{15}$.

Cette fois, donc, aucune intrusion d'une voix de poète qui vient accaparer l'attention de l'observateur. Malgré sa conscience du fait qu'il assiste à une «représentation», l'illusion d'une «scène de la vie» réussit.

Le rôle capital joué par ces deux topoï dans la réflexion contemporaine sur le théâtre semble confirmé par leur migration de la théorie française vers la critique allemande, car nous retrouvons le poète disparu et le spectateur caché dans la Hamburgische Dramaturgie, une revue de théâtre créée en 1767 par Gotthold Ephraïm Lessing dans le cadre du renouveau du Théâtre national à Hambourg. Lessing connaissait les écrits de La Motte et, de plus, il avait traduit en allemand la théorie et les pièces de Diderot (1759-1760). Il n'est guère surprenant de constater, alors, qu'à plusieurs reprises, en parlant du langage dramatique, il invoque, presque comme un automatisme, l'idée du poète trop visible. Relevant quelques remarques de Voltaire, qui s'était lamenté du fait que le public contemporain ne goûtait plus le langage poétique au théâtre et exigeait plutôt qu'on entende uniquement la voix du personnage, Lessing appuie l'opinion moderne et condamne tout dramaturge qui «an mehrern Stellen luxuriere, und seinen eignen Kopf durch die Tapete stickt ${ }^{16}{ }^{\prime}$. C'est encore à propos du théâtre de Voltaire, l'auteur dont les pièces dominent la Hamburgische Dramaturgie tout comme elles dominaient le répertoire du théâtre à Hambourg ${ }^{17}$, que Lessing fait le lien entre la disparition du

15 Ibid., t. IV, p. 1126. Sur le rôle de l'observateur / voyeur comme «posture de base» dans l'œuvre diderotienne, voir Philip Stewart, "Diderot absconditus», dans l'Encyclopédie, Diderot, l'esthétique: mélanges en hommage à Jacques Chouillet (1915-1990), textes réunis et publiés par Sylvain Auroux, Dominique Bourel et Charles Porset, Paris, Presses universitaires de France, 1991, p. 148-57; et Suzanne Pucci, «The Art, Nature and Fiction of Diderot's Beholder», Stanford French Review, 8, 1984, p. 273-294.

16 Gotthold Ephraïm Lessing, Werke, op. cit., t. IV, p. 423. Lessing écrit: «qui, s'abandonnant, ici et là, à des discours extravagants, sort la tête de derrière le décor».

17 Voir J.G. Robertson, Lessing's Dramatic Theory, being an Introduction to and Commentary 
poète et la représentation mimétique. Dans le cadre de son analyse de divers traitements de l'histoire de Mérope, Lessing évoque la première de la version voltairienne en 1743 . Il raconte qu'à la fin de la représentation le public demanda de voir l'auteur et que Voltaire parut sur la scène pour accueillir les applaudissements. Cette situation incarne les fondements de l'esthétique néoclassique rejetée par Lessing; le poète, dont les spectateurs avait senti la présence, se montre à eux pour jouir de leur admiration. Condamnant et la vanité de l'auteur et la curiosité du public, Lessing se prononce en faveur de l'illusion:

Wie schwach muss der Eindruck sein, den das Werk gemacht hat, wenn man in eben dem Augenblicke auf nichts begieriger ist, als die Figur des Meisters dagegen zu halten. Das wahre Meisterstück, dünkt mich, erfüllet uns so ganz mit sich selbst, dass wir des Urhebers darüber vergessen; dass wir es nicht als das Produkt eines einzelnen Wesens, sondern der allgemeinen Natur betrachten. ... Die Täuschung muss sehr schwach sein, man muss wenig Natur, aber desto mehr Künstelei empfinden, wenn man so neugierig nach dem Künstler ist ${ }^{18}$.

Le but d'une pièce de théâtre, et c'est l'effet que Lessing lui-même visera dans les ouvrages de sa maturité, est de s'imposer au spectateur avec la force d'un phénomène naturel, une action de la vraie vie ${ }^{19}$.

Quant à l'image du spectateur caché, Lessing ne la refond pas pour la Dramaturgie; il se contente de reproduire en allemand la plus grande partie du passage pertinent des Bijoux indiscrets de Diderot. Ce qu'il en dit dans sa présentation montre qu'il tient cet extrait pour un exposé génial des éléments essentiels de la nouvelle esthétique: le refus du formalisme, la nécessité de l'illusion et la récupération d'un vrai classicisme, celui des anciens ${ }^{20}$.

on his «Hamburgische Dramaturgie», Cambridge, Cambridge University Press, 1939; New York: B. Blom, 1965, p. 48.

18 Gotthold Ephraïm Lessing, Werke, op. cit., t. IV, p. 398-99. Lessing estime "que l'impression laissée par une œuvre doit être très faible, si au moment de son exécution on ne pense qu'à y comparer l'image du maître. Le vrai chef-d'œuvre, me semble-t-il, nous remplit tellement de lui-même que nous en oublions tout à fait l'auteur. Nous ne le prenons pas pour l'œuvre d'un particulier mais de la nature en général. [...] L'illusion doit être très faible, et on doit y sentir très peu de naturel et $d^{\prime}$ autant plus d'artifice, quand on est si curieux de connaître l'artiste.»

19 Les pièces qu'il créa pendant et après cette période sont Minna von Barnhelm (1767), Emilia Galotti (1772) et Nathan der Weise (1779).

20 «Den klaren lautern Diderot! Aber alle diese Wahrheiten waren damals in den Wind 
On peut donc conclure que les images du poète disparu et du spectateur caché ont une importance particulière pour l'esthétique théâtrale de l'époque, car elles permettent aux dramaturges de mieux exposer pour leurs lecteurs ce qu'ils veulent dire par le mot «illusion». Cette façon de concevoir l'illusion, comme un jeu du faire semblant entre un poète et un spectateur qui se dérobent l'un à l'autre, sert aussi à souligner la parenté entre le théâtre et les arts plastiques, car ces deux images correspondent très précisément à certaines tendances de la réflexion contemporaine dans ce domaine.

Selon la pensée de l'époque, l'art qui réussissait le mieux à masquer la présence de l'artiste et à créer l'illusion du réel était la peinture. En premier lieu, cette conception résulte d'une caractéristique de la perception assez généralement reconnue. Quand on regarde un tableau figuratif, on ne peut pas le constituer simultanément comme l'image de quelque chose et comme technique. Tant qu'on est occupé à reconstituer le tableau comme une image en trois dimensions représentant une scène de la vie, les traits de pinceau, les taches de couleur, la distribution du clair-obscur restent comme imperceptibles ${ }^{21}$. Regarder une peinture comme tableau ou en analyser la matérialité sont deux façons bien distinctes de la contempler. Cette capacité qu'a la peinture de tromper l'œil fut remise en valeur au cours des débats sur le coloris qui avaient préoccupé l'Académie de peinture et sculpture pendant une quarantaine d'années, de 1668 à 1708. Les nombreux écrits de Roger de Piles, chef de file des coloristes, donnèrent à ces idées une large diffusion à l'échelle européenne $^{22}$. $\mathrm{D}^{\prime}$ après de Piles, la supériorité de la peinture sur les autres arts résulte précisément du fait que «Les autres arts ne font que réveiller l'idée des choses absentes, au lieu que la peinture les supplée entière-

gesagt» (Gotthold Ephraïm Lessing, Werke, op. cit., t. IV, p. 626). On pourrait aussi démontrer que l'anecdote du spectateur caché transmet une notion de la représentation théâtrale qui cadre avec l'analyse sémiotique des arts telle que Lessing la conçoit dans son Laocoon et dans d'autres documents qui y sont reliés.

21 Cela est le point de départ de l'étude d'Ernst Gombrich, Art and Illusion: A Study in the Psychology of Pictorial Representation, New York, Bollingen Foundation, 1960, p. 5-6. Norman Bryson s'appuie sur le même principe dans son étude de la peinture de l'Ancien Régime: «An image can persuade us that it reflects the real only for as long as it effaces the traces of its own production and conceals the independent material existence of the signifier» (Word and Image: Painting of the Ancien Régime, Cambridge, Cambridge University Press, 1981, p. 27).

22 Voir Thomas Puttfarken, Roger de Piles' Theory of Art, New Haven et Londres, Yale University Press, 1985, p. 125; René Verbraeken, «Roger de Piles et le vocabulaire artistique», Études romanes, 18, 1979, p. 128. 
ment et les rend présentes par son essence qui ne consiste pas seulement à plaire aux yeux mais à les tromper ${ }^{23}$.» Le travail du peintre

imite Dieu dans sa Toutepuissance; c'est-à-dire dans la création des choses visibles. Le poète peut bien en faire la description par la force de ses paroles, mais les paroles ne seront jamais prises pour la chose même, et n'imiteront point cette Toutepuissance, qui d'abord s'est manifestée par des créatures visibles. Au lieu que la peinture avec un peu de couleurs, et comme de rien, forme et représente si bien toutes les choses qui sont sur la terre, sur les eaux, et dans les airs, que nous les croyons véritables ${ }^{24}$.

Les esthéticiens de la génération suivante évoqueront l'illusion visuelle créée par la peinture - sa vraie magie, selon de Piles et ses disciples en termes sémiotiques, accentuant la différence entre les "signes naturels» qu'emploie la peinture et les signes «artificiels» ou «arbitraires» que sont les mots. L'abbé Dubos, par exemple, note dans ses Réflexions critiques que

les signes que la peinture emploie pour nous parler ne sont pas des signes arbitraires et institués tels que sont les mots dont la poésie se sert. La peinture emploie des signes naturels, dont l'énergie ne dépend pas de l'éducation. Ils tirent leur force du rapport que la Nature elle-même a pris soin de mettre entre les objets extérieurs et nos organes, afin de procurer notre conservation. [...] Si notre esprit n'y est point trompé, nos sens du moins y sont abusés. La figure des objets, leur couleur, les reflets de la lumière, les ombres, enfin tout ce que l'œil peut apercevoir, se trouve dans un tableau comme nous le voyons dans la nature; elle se présente dans un tableau sous la même forme où nous la voyons réellement ${ }^{25}$.

23 Roger de Piles, Cours de peinture par principes, Paris, 1708, p. 41.

24 Ibid., p. 452-453. De Piles justifie l'importance qu'il accorde au coloris en insistant sur le fait que c'est sous la forme des couleurs, et non pas comme autant de contours, que le monde se présente à notre perception. Il écrit à ce propos: «Le peintre qui est un parfait imitateur de la nature, pourvu de l'habitude d'un excellent dessein, comme nous le supposons, doit donc considérer la couleur comme son objet principal, puisqu'il ne regarde cette même nature que comme imitable, qu'elle ne lui est imitable, que parce qu'elle est visible, et qu'elle n'est visible que parce qu'elle est colorée» (ibid., p. 311-312).

25 Abbé Jean-Baptiste Du Bos, Réflexions critiques sur la poésie et sur la peinture, Paris, École nationale supérieure des Beaux-arts, 1993, p. 133-134. (Cette édition est fondée sur l'édition posthume des Réflexions parue chez Pissot en 1755, qui reprenait la dernière édition corrigée et augmentée par Dubos de son vivant.) 
Ainsi, supprimer dans une pièce tout ce qui annonce l'existence du poète conférerait au théâtre les qualités tant prisées de la peinture; tous les signes théâtraux, y compris le discours, seraient convertis en signes naturels.

Il est évident que l'image du spectateur caché renforce également les affinités entre les œuvres dramatiques et les arts plastiques, mais cette fois sous l'angle de la réception de l'œuvre. Dans une étude capitale publiée en 1980, Michael Fried analyse certaines tendances de la peinture en France de la période qui va de 1750 à 1780 . Il croit déceler dans les tableaux de nombreux artistes un effort pour créer une image qui semble «nier» ou «neutraliser» la présence du spectateur devant la toile ${ }^{26}$. Les peintres en question privilégient la représentation de personnages qui se dérobent au regard du spectateur, plutôt que de le solliciter: figures endormies, mal voyantes, représentées de dos, absorbées par la lecture ou le jeu; personnages dont l'attention est accaparée par un événement qui se produit dans un ailleurs impénétrable pour le spectateur, ou bien portant des habits qui trahissent la négligence de quelqu'un qui ne se croit pas «visible». La mise en scène de ces diverses formes de ce que Fried nomme «l'absorption» fascine le public qui fréquente les salons et les ateliers. D'ailleurs, cet effet, qui transforme le spectateur en témoin ignoré, devient, dans le nouveau domaine de la critique de l'art, un des critères auxquels on fait appel pour évaluer les mérites d'une toile ${ }^{27}$. Fried insiste à plusieurs reprises sur le parallélisme évident entre la situation du spectateur placé devant ces tableaux et celle du public en train de regarder une pièce dans l'esthétique théâtrale de Diderot. Le topos du spectateur caché incarne cette tendance, et il est intéressant de constater que Paul Landois, le dramaturge chez qui elle figure d'abord, reçut une formation de peintre dans l'atelier de son père, le maître-peintre Michel Landois ${ }^{28}$.

Mais si d'un côté ces deux topoï affirment la parenté entre théâtre et peinture, ils suggèrent en même temps des rapports avec la nouvelle vision de l'univers issue des travaux scientifiques. En français, le mot «spectacle» a plus d'un sens. Dérivé du nom «spectaculum» et du verbe

26 Voir Michael Fried, Absorption and Theatricality: Painting and Beholder in the Age of Diderot, Berkeley, Los Angeles et Londres, University of California Press, 1980, p. 7-70.

27 Voir ibid., p. 8-17.

28 Voir Romira Worvill, «Recherches sur Paul Landois, collaborateur de l'Encyclopédie», Recherches sur Diderot et sur l'Encyclopédie, 23, octobre 1997, p. 127-140, particulièrement p. 131-132. 
«spectare», qui signifient respectivement en latin "chose vue» et "regarder", ce mot évoque d'abord un objet ou une scène qui s'offre aux yeux. Mais le terme "spectacle» s'emploie aussi pour désigner n'importe quelle représentation se déroulant sur les planches. Par conséquent, cette expression se prête facilement à des emplois métaphoriques qui mêlent les deux possibilités. On en trouve des exemples chez plusieurs scientifiques et philosophes influencés par la cosmologie de Copernic, Kepler et Galilée, dont les découvertes avaient imposé une révision radicale de la position des humains dans l'univers. L'affirmation que le mouvement de la Terre est une réalité physique, vécue chaque jour, avait bouleversé les vieilles certitudes, surtout l'idée de la terre comme «la scène sur laquelle s'était déroulé le drame de l'incarnation de Dieu et de la rédemption des hommes ${ }^{29}{ }^{\prime}$. Expulsés du centre qu'ils occupaient depuis des siècles, les humains se virent reléguer dans une espèce de loge, d'où ils observaient, comme au théâtre, $l^{\prime}$ immense danse des astres se renouvelant sans cesse devant et autour d'eux. En effet, le mot "spectacle», dans l'expression «spectacle de la nature» ou parfois "spectacle de l'univers», commence à revenir avec une fréquence remarquable sous la plume des philosophes de l'époque. Une recherche sur ces termes dans la base de données informatisée du projet American and French Research on the Treasury of the French Language (ARTFL) semble indiquer que cette formule acquit le statut de lieu commun au cours du XVIII ${ }^{\mathrm{e}}$ siècle et qu'elle a été utilisée, entre autres, par Fénelon, Prévost, Voltaire, Diderot, Rousseau, d'Holbach, Helvétius, Buffon et Bernardin de Saint-Pierre, soit pour évoquer les phénomènes célestes, soit pour parler du monde naturel sur la Terre ${ }^{30}$. Chose curieuse pourtant: l'expression ne figure pas

29 «The scene on which the incarnation of God and the redemption of mankind had been enacted» (E.J. Diksterhuis, The Mechanization of the World Picture: Pythagoras to Newton, traduction de C. Dikshoorn, New Jersey, Princeton University Press, 1986; Oxford, Oxford University Press, 1961, p. 382-383).

30 Pour la période 1680-1800, on peut repérer 43 occurrences de l'expression «spectacle de la nature» dans la base ARTFL, dont quatre sont des allusions au titre de l'ouvrage de l'abbé Pluche, le Spectacle de la Nature. On parlera de cette œuvre par la suite. La toute première occurrence (dans cette base de données) se trouve dans le Traité de l'existence de Dieu de Fénelon (1713), et la formule se montre particulièrement fréquente chez Diderot (sept exemples dans les textes de la base ARTFL) et chez Rousseau (six exemples dans ARTFL). Elle persiste encore au XIX ${ }^{\mathrm{e}}$ siècle (une trentaine d'occurrences dans ARTFL, dont quatre chez Flaubert), mais semble tomber en désuétude au XXe ${ }^{\mathrm{e}}$, car pour la période 1900-1964 il n'y a que quatre exemples, datant de 1925 (deux exemples), de 1926 et de 1936. Évidemment, ces statistiques ne permettent aucune conclusion définitive, vu le nombre limité de textes qui figurent 
en tant que telle dans les Entretiens sur la pluralitédes mondes de Fontenelle (1686), quoique cet ouvrage de vulgarisation scientifique (caractérisé par Paul Hazard comme «une préface, coquette et profonde, à une nouvelle interprétation de l'univers ${ }^{31} \gg$ ) semble être le premier à exploiter de façon systématique l'idée de l'univers comme spectacle et comme divertissement. Il s'agit d'une série de conversations entre un philosophe et une marquise: lui, érudit et spirituel; elle, intelligente, curieuse et aimable, mais tout à fait ignorante quant à la disposition des corps célestes et des mouvements planétaires. Au savant donc de l'éclairer, ce qu'il fait pendant qu'ils se promènent, le soir, sous les arbres du parc de la marquise. Les Entretiens s'ouvrent sur la description du spectacle qui s'y offre à leur regard émerveillé:

La lune était levée il y avait peut-être une heure, et ses rayons, qui ne venaient à nous qu'entre les branches des arbres, faisaient un agréable mélange d'un blanc fort vif, avec tout ce vert qui paraissait noir. Il n'y avait pas un nuage qui dérobât ou qui obscurcît la moindre étoile; elles étaient toutes d'un or pur et éclatant, et qui était encore relevé par le fond bleu où elles sont attachées. Ce spectacle me fit rêver... ${ }^{32}$

Cette description forme une sorte de tableau qui sert à placer le lecteur au bon endroit, c'est-à-dire devant les phénomènes que Fontenelle va expliquer, et il est intéressant de noter que l'auteur ne manque pas d'évoquer les couleurs sous lesquelles se présentent les différents éléments de l'ensemble. Dès ce premier entretien, la métaphore du spectacle ressort, explicite. Fontenelle explique à sa marquise:

Je me figure toujours que la nature est un grand spectacle, qui ressemble à celui de l'opéra. Du lieu où vous êtes à l'opéra, vous ne voyez pas le théâtre tout-à-fait comme il est: on a disposé les décorations et les machines pour faire de loin un effet agréable, et on cache à votre vue ces roues et ces contrepoids qui font tous les mouvements. Aussi ne vous embarrassez-vous guère de deviner comment tout cela joue. Il n'y a peut-être que quelque machiniste caché dans le parterre, qui s'inquiète d'un vol qui lui aura paru extraordinaire, et qui veut absolument

dans la base ARTFL; elles sont néanmoins suggestives par rapport au thème que nous développons dans cet exposé.

31 Paul Hazard, la Crise de la conscience européenne, 1680-1715, Paris, Fayard, 1961, p. 287.

32 Bernard le Bovier, sieur de Fontenelle, CFuvres complètes, édition de G.-B. Depping, Paris, 1813; Genève, Slatkine reprints, 1968, 3 tomes, t. II, p. 8. 
démêler comment ce vol a été exécuté. Vous voyez bien que ce machiniste-là est assez fait comme les philosophes ${ }^{33}$.

Le choix de l'opéra comme exemple de spectacle, tout éloigné qu'il puisse paraître des préoccupations d'un Diderot ou d'un Lessing, n'a rien d'étonnant ici, car, à l'époque où Fontenelle agitait ces questions, on tenait l'opéra pour le genre consacré à la création d'illusions ${ }^{34}$. L'opéra proposait un spectacle divertissant aux yeux des spectateurs, agréablement trompés par les effets de machines ingénieuses. L'objectif de la tragédie, par contre, était de persuader l'esprit grâce à la rigoureuse exploitation des principes de la vraisemblance, conçus comme le contraire de l'illusion plutôt que comme son complément, tel que ce sera le cas plus $\operatorname{tard}^{35}$. Le spectacle de l'univers qui en impose à nos yeux correspond naturellement pour Fontenelle aux merveilleux effets visuels procurés par l'opéra. Les images du spectacle et du spectateur se répètent tout le long de ces Entretiens. Déjà, dans la préface, l'auteur affirme «que les idées de physique [...] sont riantes d'elles-mêmes, et que dans le même temps qu'elles contentent la raison, elles donnent à l'imagination un spectacle qui lui plaît autant que s'il était fait exprès pour elle ${ }^{36}{ } »$. Plus loin, il reprend l'idée d'un "spectateur suspendu» qui regarde tourner le globe au-dessous de ses pieds et passe en revue tous les continents et les habitants du monde, tandis qu'à d'autres moments il envisage les habitants éventuels d'autres planètes comme les spectateurs de la nôtre ${ }^{37}$. En parlant du soleil, Fontenelle constate, non sans ironie, qu' «il n'y a qu'un lieu dans le monde, d'où l'étude des astres puisse être extrêmement facile; et justement, dans ce lieu-là, il n'y a personne ${ }^{38} »$. Néanmoins, il se dit reconnaissant envers Copernic «d'avoir rabattu la vanité des hommes, qui s'étaient mis à la plus belle place de l'univers ${ }^{39}{ }^{\prime}$, et il insiste beaucoup sur notre rôle de simples témoins devant une nature indifférente, «qui n'entre point en connaissance de tous ces petits mouve-

33 Ibid., t. II, p. 10.

34 Voir Marion Hobson, The Object of Art: The Theory of Illusion in Eighteenth-Century France, Cambridge, Cambridge University Press, 1982, p. 36.

35 Voir ibid., p. 42.

36 Fontenelle, CEuvres complètes, op. cit., t. II, p. 5.

37 Ibid., t. II, p. 19. Fontenelle utilise l'expression «le spectateur suspendu» dans une lettre de réponse à l'un de ses critiques (ibid., t. II, p. 82).

38 Ibid., t. II, p. 50.

39 Ibid., t. II, p. 15. 
ments particuliers [et] nous emporte tous ensemble d'un mouvement

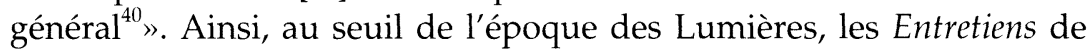
Fontenelle exposent d'une façon très claire la nouvelle position de l'être humain vis-à-vis de l'univers, le définissant comme spectateur.

Le thème de la nature comme spectacle est développé encore par Noèl Antoine Pluche dans son ouvrage populaire le Spectacle de la nature, ou, Entretiens sur les particularités de l'histoire naturelle, qui ont paru les plus propres à rendre les jeunes-gens curieux et à leur former l'esprit, qui commença à paraître en 1732. C'est le monde de la nature sur Terre, plutôt que les corps célestes, qui retient l'attention de Pluche, mais son approche s'inspire directement de celle de Fontenelle. Comme chez ce dernier, son texte prend la forme d'une série d'entretiens, chacun consacré à un phénomène naturel, tels les abeilles, les vers à soie, les chenilles, les plantes etc.. Les conversations se passent parmi un groupe de personnages réunis à la campagne chez $\mathrm{M}$. le comte de Jonval, et Pluche souligne l'à-propos de ce décor "pour le lieu de la scène d'un Dialogue sur l'Histoire de la Nature ${ }^{41} \gg$. Il explique dans sa préface qu'il a rejeté l'idée de présenter des échanges imaginaires entre scientifiques morts (allusion probable aux Dialogues des morts de Fontenelle), préférant à de tels spécialistes des personnages avec qui les jeunes lecteurs pourront plus facilement s'identifier. C'est donc un jeune homme de qualité, le chevalier du Breuil, qui profite de ces discussions avec le comte de Jonval lui-même, Mme la comtesse et le prieur du lieu, dont le rôle est de marquer les bornes de la raison humaine et de contrôler les conclusions par rapport aux articles de foi. Au début du deuxième entretien, le chevalier, qui avait eu l'idée de résumer par écrit l'essentiel de la conversation de la veille, se voit attribuer la fonction de secrétaire, ce qui entraîne une allusion directe à Fontenelle: «Monsieur le Chevalier, puisque vous savez déjà penser vous-même, \& donner de la netteté et des grâces aux pensées des autres, voilà qui est fait, vous serez le Fontenelle de notre Académie ${ }^{42}$.» Mais ce n'est pas uniquement en raison de la forme que cet ouvrage se situe dans la tradition inaugurée par l'illustre académicien. La préface de Pluche révèle qu'il a adopté très consciemment l'orientation de spectateur élaborée par son prédécesseur. Deux

40 Ibid., t. II, p. 20.

41 Antoine Noèl Pluche, le Spectacle de la Nature, ou, Entretiens sur les particularités de l'histoire naturelle, qui ont paru les plus propres à rendre les jeunes-gens curieux et à leur former l'esprit, La Haye, 1732-1735; 1743-1753, 8 tomes, t. I, p. xiii.

42 Ibid., t. I, p. 36. 
métaphores clés dominent la pensée de Pluche dans cette préface, et le passage de l'une à l'autre incarne très bien l'évolution qu'a subie la posture de l'être humain vis-à-vis de la nature. Pluche commence par envisager une nature qui nous parle et qui se manifeste sous la forme d'un texte:

Tous les corps qui nous environnent, les plus petits comme les plus grands, nous apprennent quelques vérités: ils ont tous un langage qui s'adresse à nous, même qui ne s'adresse qu'à nous. Leur structure particulière nous dit quelque chose. Leur tendance à une fin, nous marque l'intention de l'Ouvrier. Leurs rapports entre eux \& avec nous, sont autant de voix distinctes qui nous appellent, qui nous offrent des services; et qui, par les avis qu'elles nous donnent, remplissent notre vie de commodités, notre esprit de vérités, notre cœur de reconnaissance. Enfin on peut dire que la Nature est le plus savant \& le plus parfait de tous les Livres propres à cultiver notre Raison, puisqu'il renferme à la fois les objets de toutes les Sciences, et que l'intelligence n'en est bornée ni à aucune langue, ni à aucunes personnes ${ }^{43}$.

Mais ces voix et ce texte qui s'adressent à l'oreille et à l'esprit se transforment tout d'un coup en un objet de la vue:

C'est de ce Livre, exposé à tous les yeux, \& cependant assez peu lu, que nous entreprenons, pour ainsi dire, de donner un extrait, dans le dessein de faire connoître aux jeunes Lecteurs des richesses qu'ils possédoient sans en jouir, \& de rapprocher sous leurs yeux ce que l'éloignement, la petitesse, \& l'inattention leur déroboient.

De cette image de la nature comme livre en tant qu'objet de la vue, Pluche passe facilement à l'idée de la nature comme spectacle tout court, en s'appuyant très visiblement sur la métaphore théâtrale des Entretiens de Fontenelle:

Nous jouissons tous de la vue et des dehors de la Nature. Le Spectacle est pour nous. En nous y bornant, nous découvrons suffisamment de toute part le beau, l'utile \& le vrai. [...] Mais prétendre pénétrer le fond même de la Nature, vouloir rappeller les effets à leurs causes spéciales, vouloir comprendre l'artifice \& le jeu des ressorts, \& les plus petits élémens dont ces ressorts sont composés, c'est une entreprise hardie \& d'un succès trop incertain. Nous la laissons à ces génies d'un 
ordre supérieur, à qui il peut avoir été donné d'entrer dans ces mystéres \& de voir. Pour nous, nous croyons qu'il nous convient mieux de nous en tenir à la décoration extérieure de ce Monde, \& à l'effet des machines qui forment le Spectacle. Nous y sommes admis. On voit bien même qu'il n'a été rendu si brillant que pour piquer notre curiosité. Mais, contens d'une représentation qui remplit suffisamment nos sens \& notre esprit, il n'est pas nécessaire de demander que la salle des machines nous soit ouverte ${ }^{44}$.

D'ailleurs, Pluche utilise un mot assez révélateur pour décrire les scientifiques dont il parle dans sa préface: il les nomme «observateurs». Ainsi, son texte, qui dirige notre regard vers les merveilles de la nature sur terre, fait pendant à l'exposé des phénomènes célestes par Fontenelle. Tous les deux, ils nous situent comme spectateurs par rapport aux deux sphères qui les intéressent et qui, ensemble, englobent la nature entière. Cette orientation visuelle envers le monde, entraînée par la quête scientifique et renforcée par l'emploi d'outils tels le télescope et le microscope qui transformèrent les possibilités de la vue, finit par faire partie intégrante de la définition de l'être humain. "L'homme est né spectateur», affirme Jaucourt, citant Batteux, dans son article «Spectacles» de l'Encyclopédie; «l'appareil de tout l'univers que le Créateur semble étaler pour être vu et admiré, nous le dit assez clairement».

En France, la principale réforme à toucher le théâtre de cette époque, la suppression des banquettes sur la scène de la Comédie-Française pour en éliminer un public dont la présence commence à déranger, semble faire écho à ces révolutions scientifiques et métaphysiques ${ }^{45}$. La pour-

44 Ibid., t. I, p. viii-x. On dirait donc que, dans la préface de Pluche, on voit disparaître la mentalité d'une époque révolue. La métaphore du livre rappelle la description que donne Michel Foucault de l'épistémè d'avant l'ère scientifique, mais cette métaphore-là cède la place à celle du spectacle. Foucault caractérise l'ancienne épistémè de la façon suivante: «Il n'y pas de différence entre [les] marques visibles que Dieu a déposées sur la surface de la terre pour nous en faire connaitre les secrets intérieurs, et les mots lisibles que l'écriture, ou les sages de l'Antiquité, qui ont été éclairés par une divine lumière, ont déposés en ces livres que la tradition a sauvés. Le rapport aux textes est de même nature que le rapport aux choses; ici et là, ce sont des signes qu'on relève. [...] La vérité de toutes ces marques - qu'elles traversent la nature ou qu'elles s'alignent sur les parchemins et dans les bibliothèques - est partout la même: aussi archaïque que l'institution de Dieu. [...] Il n'y a partout que le même jeu, celui du signe et du similaire, et c'est pourquoi la nature et le verbe peuvent s'entrecroiser à l'infini, formant pour qui sait lire un grand texte unique» (les Mots et les choses: une archéologie des sciences humaines, Paris, Gallimard, 1966, p. 48-49).

45 Cette réforme eut enfin lieu en 1759, après une longue lutte de la part de critiques et de dramaturges. Voir, à ce propos, Paul Berret, «Comment la scène de théâtre au 
suite de l'illusion scénique, que la scène ainsi libérée allait favoriser, en fait aussi partie sans doute. La disparition du poète dont la signature n'est plus lisible dans son œuvre, ainsi que la solitude du spectateur installé devant une action qui se déroule sans intervention de sa part, semblent exprimer à la fois la nouvelle façon de concevoir une pièce de théâtre et la situation de l'être humain dans un univers révélé non pas par un texte sacré mais par les observations scientifiques. Diderot, Lessing et leurs confrères ne cherchaient peut-être rien d'autre qu'à retrouver l'harmonie perdue entre le système du théâtre et le système du monde. Ils disaient à leurs contemporains, comme Fontenelle à sa marquise, «je crois que je n'ai qu'à tirer le rideau, et à vous montrer le monde ${ }^{46} »$.

ROMIRA WORVILL

Acadia University

XVIII ${ }^{\mathrm{e}}$ siècle a été débarrassée de la présence des Gentilshommes", Revue d'histoire littéraire de la France, 8, 1901, p. 456; et Barbara G. Mittman, Spectators on the Paris Stage in the Seventeenth and Eighteenth Centuries, Ann Arbor, Michigan, UMI Research Press, 1984.

46 CEuvres complètes, op. cit., t. II, p. 11. 